\title{
ETCE2002/CAE-29010
}

\section{LINEAR TEMPORAL STABILITY ANALYSIS OF A LOW-DENSITY ROUND GAS JET INJECTED INTO A HIGH-DENSITY GAS}

\author{
Anthony L. Lawson \\ School of Aerospace and Mechanical Engineering \\ The University of Oklahoma \\ Norman, Oklahoma
}

\author{
Ramkumar N. Parthasarathy \\ School of Aerospace and Mechanical Engineering \\ The University of Oklahoma \\ Norman, Oklahoma \\ rparthasarathy@ou.edu
}

\begin{abstract}
It has been observed in previous experimental studies that round helium jets injected into air display a repetitive structure for a long distance, somewhat similar to the buoyancy-induced flickering observed in diffusion flames. In order to investigate the influence of gravity on the near-injector development of the flow, a linear temporal stability analysis of a round helium jet injected into air was performed. The flow was assumed to be isothermal and locally parallel; viscous and diffusive effects were ignored. The variables were represented as the sum of the mean value and a normal-mode small disturbance. An ordinary differential equation governing the amplitude of the pressure disturbance was derived. The velocity and density profiles in the shear layer, and the Froude number (signifying the effects of gravity) were the three important parameters in this equation. Together with the boundary conditions, an eigenvalue problem was formulated. Assuming that the velocity and density profiles in the shear layer to be represented by hyperbolic tangent functions, the eigenvalue problem was solved for various values of Froude number. The temporal growth rates and the phase velocity of the disturbances were obtained. The temporal growth rates of the disturbances increased as the Froude number was reduced (i.e. gravitational effects increased), indicating the destabilizing role played by gravity.
\end{abstract}

\section{NOMENCLATURE}

\section{English Symbols}

\begin{tabular}{ll}
\hline$d$ & jet diameter \\
$D_{b}$ & binary diffusivity coefficient
\end{tabular}

Froude number; $F r^{2}=\frac{U_{j}^{2}}{g R} \frac{\rho_{j}}{\left(\rho_{\infty}-\rho_{j}\right)}$ acceleration due to gravity non-dimensional coefficient of the buoyancy term in the pressure disturbance equation;

$$
G=\frac{1}{F r^{2} \frac{R}{\theta}\left(\frac{\rho_{\infty}}{\rho_{j}}-1\right)}
$$<smiles>C1[AlH][AlH]1</smiles>

wavenumber

azimuthal wavenumber

pressure

radial coordinate

low-density jet exit radius

radius of the jet shear layer

Density ratio

Schmidt number; $S c=\frac{v}{D_{b}}$

time

jet axial velocity component

jet base velocity

jet base centerline velocity

jet radial velocity component

jet azimuthal velocity component

axial coordinate

local mass fraction of injected gas

\section{Greek Symbols}

$\begin{array}{ll}\delta_{i j} & \text { Kronecker delta } \\ \nabla & \text { del operator } \\ \phi & \text { azimuthal coordinate }\end{array}$




$\begin{array}{ll}\lambda & \text { wavelength } \\ \mu & \text { fluid viscosity } \\ v & \text { fluid kinematic viscosity } \\ \rho & \text { fluid mass density } \\ \rho_{j} & \text { fluid mass density of low-density gas } \\ \rho_{\infty} & \text { fluid mass density of high-density ambient } \\ \theta & \text { gas } \\ \Omega & \text { shear layer momentum thickness } \\ & \text { angular frequency }\end{array}$

\section{Superscripts}

\section{( )' fluctuations}

( ) averaged variable

$(\wedge) \quad$ amplitude of variable

( ) dimensional variable

\section{Subscripts}

\begin{tabular}{ll}
\hline$\infty$ & ambient gas property \\
$j$ & low-density gas property
\end{tabular}

\section{INTRODUCTION}

Low-density gas jets injected into higher density ambient gases are encountered in many engineering and technical applications such as plumes of diffusion flames, fuel leaks, engine and industry exhaust, and in natural phenomena such as fires and volcanic eruptions. Recent experimental studies (Monkewitz et al, 1990; Subbarao and Cantwell, 1992; Kyle and Sreenivasan, 1993; Cetegen and Kasper, 1996) indicate that at certain conditions, low-density gas jets injected into high-density gases may sustain an absolute instability leading to highly periodic oscillations. This phenomenon is similar to the buoyancy-induced flickering observed in diffusion flames (Buckmaster and Peters, 1986; Chen et al., 1988; Cetegen and Dong, 2000). It is thus plausible that the periodicity observed in the oscillations of low-density gas jets injected into a highdensity medium is related to buoyancy effects. Therefore, an investigation of the influence of gravity on the disturbances in the near-injector region of low-density jets is necessary. This study aims to highlight as well as improve the understanding of the nature of flow instability in low-density round gas jets injected into high-density gases.

Round jets with homogeneous shear layers have been studied extensively in the past. The near-injector region of a round jet, as influenced by disturbances, has a direct influence on the flow development in the far-field of the jet. Early studies of jet instability were reviewed by Michalke (1984). These studies were carried out to understand the transition from laminar flow to turbulence and to describe the evolution of the large-scale coherent structures in the near-injector field. The critical Reynolds number denoting the transition from laminar to turbulent flow was documented. Also, it was found that the streak line patterns obtained using the results of the linear stability analysis were in good agreement with the first-stage of the vortex rolling-up process that was observed experimentally. Cohen and Wygnanski (1987) concluded that the linear stability analysis was able to correctly predict the local distribution of amplitudes and phases in an axisymmetric jet that was excited by external means.

Monkewitz and Sohn (1988) re-examined the linear inviscid stability analysis of compressible heated axisymmetric jets with particular attention to the impulse response of the flow, using assumed forms of the velocity and density profiles in the shear layer. Two different responses were identified based on the results. In one case, the flow was absolutely unstable, when a locally generated small disturbance grew exponentially at the site of the disturbance and eventually affected the entire flow region. In the other case termed convective instability, the disturbance was convected downstream leaving the mean flow undisturbed. Monkewitz and Sohn (1988) documented the boundaries between the absolute and convective instability assuming locally parallel flow, infinite Froude number, and zero Eckert number. It was shown that heated (low-density) jets injected into ambient gas of high density developed an absolute instability and became self-excited when the jet density was less than 0.72 times the ambient gas density. Yu (1990) carried out a similar analysis for two-dimensional inertial jets and wakes with non-uniform density and axial velocity profiles neglecting gravitational effects. It was found that the absolute frequencies and wavenumbers of small disturbances in the near-injector region scaled with the jet/wake width. Jendoubi and Strykowski (1994) considered the stability of axisymmetric jets with external co-flow and counterflow. The boundaries between absolute and convective instability were distinguished for various parameters: jet-to-ambient velocity ratio, density ratio, jet Mach number, and the shear layer thickness. The onset of global self-excitation identified in laboratory jets agreed reasonably well with the predictions.

Kyle and Sreenivasan (1993) performed experiments at low Richardson numbers to study the instability and breakdown of axisymmetric helium/air mixtures emerging into ambient air using high-speed photography and velocity measurements. An intense oscillating instability was observed when the ratio of the jet exit density to the ambient fluid density was less than 0.6. Raynal et al. (1996) carried out experiments with variable-density plane jets issuing into ambient air for a range of density ratios ( 0.14 to 1$)$ and a range of Reynolds numbers (250 to 3000). It was found that when the jet to ambient fluid density ratio was less than 0.7 , the jets exhibited self-excited oscillations. Richards et al. (1996) used Mie scattering to visualize helium-air jets injected into air. Intense mixing and vortex interactions characterized the selfexcited helium jets at a density ratio of 0.14 .

Note that gravitational effects have been neglected in all of the analyses listed above. The neglect of gravitational effects has been justified by the authors because of the small magnitude of the Richardson number based on the jet velocity 
and jet diameter. However, within the shear layer where the density and velocity change from jet to ambient values, the gravitational effects may not be negligible. Literature on the effects of gravity and/or buoyancy on the stability characteristics of low-density gas jets injected in higher density ambient gases is limited.

Hamins et al. (1992) used a shadowgraph technique to observe the near-field behavior of a non-reacting buoyant helium plume discharged from a round tube into air and obtained a complex relation between the Strouhal number and the Froude number. Subbarao and Cantwell (1992) performed experiments on a co-flowing buoyant jet to study the scaling properties and effects of Richardson number and Reynolds number, independently, on the natural frequency of the jet. The Strouhal number was plotted as a function of the square root of the Richardson number and was separated into three regimes. At low Richardson numbers, the flow Strouhal number scaled with an inertial timescale, while at high Richardson numbers the Strouhal number scaled with a buoyancy timescale. Between the Richardson numbers of 0.7 and 1, a transition regime occurred. Cetegen and Kasper (1996) performed experiments on the oscillatory behavior of axisymmetric buoyant plumes of helium and helium-air mixtures. The effects of varying nozzle diameters, source velocities and plume densities were investigated. Cetegen (1997-1) investigated the effect of sinusoidal forcing on an axisymmetric buoyant plume of helium and helium-air mixtures. Mushroom-shaped smallscale vortex pairs were observed in the early part of the forced plumes that were not observed in unforced plumes. Cetegen (1997-2) used digital particle image velocimetry to measure the velocity field of a naturally pulsating plume of helium-air mixture in the presence of co-flowing air. The oscillation frequency of the plume, observed using a movie camera at a frame rate of $30 \mathrm{fps}$, was between 3 and $4.5 \mathrm{~Hz}$. Pasumarthi (2000) conducted experiments to investigate the flow structure of a pulsating helium jet injected into air using quantitative rainbow schlieren deflectometry. It was found that the Richardson number had a more significant effect on the flow structure than the jet exit Reynolds number.

The present study extends the work of Buckmaster and Peters (1986) to consider the effects of gravity on the instability of a low-density gas injected into a high-density ambient. The specific objectives of the study were to perform a linear temporal stability analysis of low-density round jets injected into high-density ambient gases, and to determine the effects of buoyancy on the instabilities of the jet.

\section{THEORY}

The present analysis considers a round jet of lowdensity gas discharged into a high-density ambient gas medium. The basic jet flow is assumed to be a locally inviscid parallel flow with an axial velocity component varying radially. In order to simplify the analysis, the flow is assumed to be isothermal. The radial velocity of the jet is considered small and, therefore, neglected. An analysis considering the effects of buoyancy, along the lines of Buckmaster and Peters (1986) is performed. The analysis differs from that of Buckmaster and Peters (1986) in several ways. First, the near-injector velocity and density profiles are used in the analysis. Second, an axisymmetric configuration is studied.

\section{Linear stability analysis}

Consider a round jet (radius $R$ ) of a low-density gas (density $\rho_{j}$ ) injected vertically upward with velocity $U_{j}$ into an ambient quiescent gas of density $\rho_{\infty}$ at atmospheric pressure as illustrated in the schematic diagram of Figure 1. Representing the velocity components by $(\tilde{u}, \tilde{v}, \tilde{w})$, in cylindrical coordinates $(\tilde{x}, \tilde{r}, \phi)$ centered at the origin of the jet, and the pressure by $p$ the conservation equations governing the flow are:

$$
\begin{gathered}
\frac{\partial \tilde{\rho}}{\partial t}+\frac{\partial(\tilde{\rho} \tilde{u})}{\partial \tilde{x}}+\frac{1}{\tilde{r}} \frac{\partial(\tilde{r} \tilde{\rho} \tilde{v})}{\partial \tilde{r}}+\frac{1}{\tilde{r}} \frac{\partial(\tilde{\rho} \tilde{w})}{\partial \phi}=0 \\
\tilde{\rho}\left[\frac{\partial \vec{v}}{\partial t}+\vec{v} \bullet \nabla \vec{v}\right]=-\nabla p+g\left(\rho_{\infty}-\tilde{\rho}\right) \delta_{i 1}+\nabla \bullet(\mu \nabla \vec{v})
\end{gathered}
$$

where $g$ is the acceleration due to gravity and $\delta_{i l}$ is the Kronecker delta function with $i=1$ representing the axial direction. The binary mass diffusion equation for the diffusion of the injected gas in the ambient gas medium is given in terms of the local mass fraction of the injected gas, $Y_{j}$ (Gebhart, 1993):

$$
\tilde{\rho}\left[\frac{\partial Y_{j}}{\partial t}+\vec{v} \bullet \nabla Y_{j}\right]=\nabla \bullet\left[\tilde{\rho} D_{b} \nabla Y_{j}\right]
$$

where $D_{b}$ is the binary diffusivity coefficient.

The flow is assumed to be locally parallel, and the variables are represented as the sum of the base state value and a fluctuation:

$$
\begin{aligned}
& \tilde{u}=\bar{u}(\tilde{r})+u^{\prime} \\
& \tilde{v}=v^{\prime} \\
& \tilde{w}=w^{\prime} \\
& \tilde{p}=p^{\prime} \\
& \tilde{p}=\bar{p}(\tilde{r})+p^{\prime}
\end{aligned}
$$

Substitution of equations (4) into equations (1) and (2), linearization, and the neglect of viscous and diffusive terms yields

$$
\begin{aligned}
& \frac{\partial \rho^{\prime}}{\partial t}+\bar{u} \frac{\partial \rho^{\prime}}{\partial \tilde{x}}+\bar{\rho} \frac{\partial u^{\prime}}{\partial \tilde{x}}+\frac{1}{\tilde{r}} \frac{\partial\left(\tilde{r} \bar{\rho} v^{\prime}\right)}{\partial \tilde{r}}+\frac{\bar{\rho}}{\tilde{r}} \frac{\partial w^{\prime}}{\partial \phi}=0 \\
& \bar{\rho}\left[\frac{\partial u^{\prime}}{\partial t}+\bar{u} \frac{\partial u^{\prime}}{\partial \tilde{x}}+v^{\prime} \frac{d \bar{u}}{d \tilde{r}}\right]=-\frac{\partial p^{\prime}}{\partial \tilde{x}}-g \rho^{\prime} \\
& \bar{\rho}\left[\frac{\partial v^{\prime}}{\partial t}+\bar{u} \frac{\partial v^{\prime}}{\partial \tilde{x}}\right]=-\frac{\partial p^{\prime}}{\partial \tilde{r}} \\
& \bar{\rho}\left[\frac{\partial w^{\prime}}{\partial t}+\bar{u} \frac{\partial w^{\prime}}{\partial \tilde{x}}\right]=-\frac{1}{\tilde{r}} \frac{\partial p^{\prime}}{\partial \phi}
\end{aligned}
$$



by

The density of the mixture is related to the mass fraction

$$
\tilde{\rho}=\frac{\rho_{j}}{Y_{j}+\left(1-Y_{j}\right) S}
$$

where $\rho_{j}$ is the density of the injected gas and $\mathrm{S}$ is the density ratio $\rho_{j} / \rho_{\infty}\left(\rho_{\infty}\right.$ is the density of the ambient gas medium). Rewriting $Y_{j}$ in terms of $\rho$, we have

$$
Y_{j}=\frac{1}{1-S}\left(\frac{\rho_{j}}{\tilde{\rho}}-S\right)
$$

where, $\mathrm{S}$ is the ratio of the density of the injected fluid to that of the ambient fluid. Substituting the above expression in equation (3), linearizing, and neglecting the diffusive term, we obtain

$$
\frac{\partial \rho^{\prime}}{\partial t}+\bar{u} \frac{\partial \rho^{\prime}}{\partial \tilde{x}}+v^{\prime} \frac{d \bar{\rho}}{d \tilde{r}}=0
$$

Using equation (10), equation (5) reduces to

$$
\bar{\rho}\left[\frac{\partial u^{\prime}}{\partial \tilde{x}}+\frac{1}{\tilde{r}} \frac{\partial\left(\tilde{r} v^{\prime}\right)}{\partial \tilde{x}}+\frac{1}{\tilde{r}} \frac{d w^{\prime}}{d \phi}\right]=0
$$

Normal mode disturbances are assumed given by

$$
\left(u^{\prime}, v^{\prime}, w^{\prime}, p^{\prime}, \rho^{\prime}\right)=[\hat{u}(\tilde{r}), \hat{v}(\tilde{r}), \hat{w}(\tilde{r}), \hat{p}(\tilde{r}), \hat{\rho}(\tilde{r})] e^{i(\tilde{r} \tilde{r}-\tilde{\Omega} t+m \phi)}
$$

where $i=\sqrt{-1} ; \hat{u}, \hat{v}, \hat{w}, \hat{p}, \hat{\rho}$ are the amplitudes of the disturbances; $\tilde{k}$ is the wavenumber; $\tilde{\Omega}$ is the frequency, and $m$ is the azimuthal wavenumber. For temporal linear stability analysis, the wavenumber, $\tilde{k}$, is real while the frequency $\tilde{\Omega}=\tilde{\Omega}_{r}+i \tilde{\Omega}_{i}$ is complex. The real part $\tilde{\Omega}_{r}$ is proportional to the disturbance frequency and the imaginary part $\tilde{\Omega}_{i}$ is the temporal growth rate of the disturbance. The disturbance gets amplified if $\tilde{\Omega}_{i}$ is positive. The ratio of $\tilde{\Omega}_{r}$ to $k$ represents the wave speed, $c_{p h}$ of the disturbance.

Substituting the expressions (12) in equations (6), (7), (8), (10) and (11), and manipulating the expressions, we obtain the following equation for the pressure disturbance:

$$
\frac{d^{2} \hat{p}}{d \tilde{r}^{2}}+\left[\frac{1}{\tilde{r}}-\frac{2 \frac{d \bar{u}}{d \tilde{r}}}{\bar{u}-\frac{\tilde{\Omega}}{\tilde{k}}}-\frac{1}{\bar{\rho}} \frac{d \bar{\rho}}{d \tilde{r}}\left(1+\frac{i g}{\tilde{k}\left(\bar{u}-\frac{\tilde{\Omega}}{\tilde{k}}\right)^{2}}\right] \frac{d \hat{p}}{d \tilde{r}}-\left(\tilde{k}^{2}+\frac{m^{2}}{\tilde{r}^{2}}\right) \hat{p}=0\right.
$$

The variables are normalized using $\rho_{\infty}, \mathrm{U}_{\mathrm{j}}$, and $\theta$ (the boundary layer momentum thickness). The nondimensionalized pressure disturbance equation for the varicose mode $(\mathrm{m}=0)$ is

$$
\frac{d^{2} P}{d r^{2}}+\left[\frac{1}{r}-\frac{2 \frac{d U}{d r}}{U-\frac{\Omega}{k}}-\frac{1}{\rho} \frac{d \rho}{d r}\left(1+i \frac{1}{\operatorname{Fr}^{2} \frac{R}{\theta}\left(\frac{\rho_{\infty}}{\rho_{j}}-1\right) k\left(U-\frac{\Omega}{k}\right)^{2}}\right)\right] \frac{d P}{d r}-k^{2} P=0
$$

It should be noted that with no density gradient equation (14) reduces to the equation governing the instability of constant-density jets (Michalke, 1984). Also, as $r \rightarrow \infty$, and if gravitational effects are neglected, equation (25) is identical to the equation used by Yu and Monkewitz (1990) and Raynal et al. (1996) in the analysis of plane jets. Inclusion of buoyancy effects indicates that the stability characteristics are altered by the additional term present in equation (14).

The pressure disturbance must vanish at large radial distances from the jet and the pressure disturbance is finite at the jet axis $(\mathrm{r}=0)$ therefore

$$
\begin{aligned}
& P(\infty) \rightarrow 0 \\
& P(0) \text { is finite } \\
& \frac{d P(0)}{d r}=0 \text { due to symmetry }
\end{aligned}
$$

An eigenvalue problem is posed, for specified mean velocity and density profiles, by equation (12) along with the boundary conditions (15). Following Monkewitz and Sohn (1988), the base axial velocity and the density profiles are specified as a function of the local jet radius. The basic jet velocity profile, $U(r)$, is given by Michalke and Hermann (1982),

$$
\frac{\bar{u}(\tilde{r})-U_{\infty}}{U_{j}-U_{\infty}}=0.5\left\{1-\tanh \left[0.25 \frac{R}{\theta}\left(\frac{\tilde{r}}{R}-\frac{R}{\tilde{r}}\right)\right]\right\}
$$

where $U_{j}$ is the injected jet uniform velocity and $U_{\infty}$ is the uniform co-flow velocity. For this study the co-flow velocity was set to zero for a quiescent ambient gas and $R$ is the radial distance of the center of the shear layer. $R / \theta$ is the jet parameter that characterizes the jet velocity profile at various axial positions. The values of the jet parameter used in this study are 10 and 5 corresponding to $\tilde{x} /(2 R)=1$ and 2.67 , respectively. This is within the near-injector region of the jet and allows for the effects of buoyancy to manifest as the jet proceeds downstream (Subbarao and Cantwell, 1992). Figure 2 illustrates the basic jet velocity profiles at these two locations. The shear layer widens as the jet proceeds downstream from $R / \theta=10$ to $R / \theta=5$ indicating the downstream development of the jet. A hyperbolic tangent profile is also assumed for the basic density profile in the shear layer,

$$
\frac{\bar{\rho}(r)}{\rho_{\infty}}=1+\left(\frac{\rho_{j}}{\rho_{\infty}}-1\right)\left\{0.5\left(1-\tanh \left[0.25 \frac{R}{\theta}\left(\frac{\tilde{r}}{R}-\frac{R}{\tilde{r}}\right)\right]\right)\right\}
$$

Figure 3 illustrates the density profiles at $\mathrm{R} / \theta=10$ for $\rho_{j} / \rho_{\infty}=$ $1,0.6$, and 0.14 .

A fourth-order Runge-Kutta scheme with automatic step-size control is used to integrate the equation (14). The infinite integration domain, $0<r<\infty$ is divided into two finite domains: an inner domain $R \geq r \geq 0$ and an outer domain $r_{\infty} \geq r$ $\geq R$, where $R$ is the jet radius and $r_{\infty}$ is a specified large radius where the gradients of the velocity and density are small. A shooting method is used to determine $\Omega(k)$ such that both $\mathrm{P}$ and $\frac{d P}{d r}$ are continuous at $r=R$. A parabolic complex zero-search 
procedure was used to vary $\Omega$ for a specified $k$ until the matching conditions were satisfied to a minimum accuracy within $10^{-10}$.

\section{RESULTS AND DISCUSSION}

The near-injector region was investigated at axial locations along the jet represented by the jet parameter $(R / \theta)$ values of $10\left(\right.$ corresponding to $\left.\frac{x}{2 R}=1\right)$ and $5\left(\frac{x}{2 R}=2.67\right)$, the value of 10 being closer to the injector exit. The density ratio, $\mathrm{S}$, was varied from 1 (homogeneous shear-layer) to 0.14 (helium jet discharged into air), while varying the Froude number from infinity (no buoyancy) to 1 .

The imaginary part of the complex frequency, $\Omega_{i}$, is the temporal growth rate of the amplitude of the disturbance wave. The wavenumber $\mathrm{k}$ is the reciprocal of the wavelength. The temporal growth rate, $\Omega_{i}$, the phase velocity, $c_{p h}$, and the wavenumber $k$ were normalized using the shear-layer momentum thickness $\theta$ and the jet exit velocity $U_{j}$.

\section{Effect of density ratio}

In order to investigate the effect of an inhomogeneous shear layer only on the evolution of the jet instabilities with time, the Froude number was fixed at infinity (negligible gravity effect) and the density ratio (S) was varied from 1 to 0.14 . The variation of the normalized temporal growth rate of the instabilities with the normalized wavenumber at $R / \theta=10$ is presented in Figure 4. As the density ratio is reduced from 1 to 0.14 , the range of unstable wavenumbers is increased by about $20 \%$. This implies that the range of unstable wavelengths decreases when the gas jet density is reduced relative to the ambient gas density. The values of the temporal growth rates are also increased. The maximum growth rate is increased by about $21 \%$, as the density ratio is decreased from 1 to 0.14 . A decrease in the jet density results in the steepening of the density gradient in the shear layer, which leads to additional generation of vorticity, thus causing an increase in the disturbance growth rate. Also, the maximum growth rate occurs at a higher wavenumber with a decrease in the density ratio. The disturbance wave with the maximum growth rate is usually assumed to dominate the flow and so will be the disturbance that is observed in experiments. Thus, the above results imply that the disturbance wavelength that is observed in experiments is reduced (by about $8 \%$ ) in the presence of a higher density ambient gas. Trends similar to those observed at $R / \theta=10$ were observed at other axial locations.

Figure 5 depicts the variation of the phase velocity of the disturbances with wavenumber at $R / \theta=10$. The phase velocity of the disturbance decreases with increasing wavenumber, indicating that the disturbances move with a velocity less than the jet velocity. Also, as the density ratio is reduced, the phase velocity decreases at all unstable wavenumbers. This also implies that the range of unstable frequencies increases as the density ratio is reduced; i.e., the frequency spectrum of the fluctuations now contains additional frequencies.

\section{Effect of buoyancy}

To study the effect of buoyancy on the jet temporal instability, the density ratio was fixed at 0.14 (corresponding to a helium jet injected into air), while the Froude number was varied from infinity (negligible buoyancy) to 1. Figures 6 and 7 display the variation of the temporal growth rates with wavenumber at $R / \theta=10$ and 5 respectively, as the Froude number (Fr) is reduced from infinity to 1 . The temporal growth rates for the constant-density jet are included as a baseline for comparison. At all Froude numbers, the temporal growth rates exceed those of the constant-density jet at both $R / \theta=10$ and 5 . Also, the wavenumber corresponding to the maximum growth rates exceed those of the constant-density jet, even though this appears to be due to the change in density ratio. When the effect of changing the Froude number alone is considered, no significant change in the wavenumber corresponding to the maximum growth rate is observed, except for $\mathrm{Fr}=1$ at $R / \theta=5$. Thus, it seems that gravity does not have a "significant" effect on the wavenumber corresponding to the maximum growth rate at $\mathrm{Fr} \geq 1$.

In Figure 6 , at $R / \theta=10$, as the Froude number is reduced from infinity to 5 , no significant change is observed in the values of the temporal growth rates. The growth rates are increased, though, as the Froude number is reduced further. The maximum growth rate increases by about $4.4 \%$, when the Froude number is reduced from infinity to 2 , but the range of unstable wavenumbers is not altered. At a Froude number of unity, the maximum growth rate is increased by about $18 \%$, while the range of unstable wavenumbers is increased by a mere $3.4 \%$. Results for various Froude numbers at a downstream location, are given in Figures 7 and $9 . \quad \mathrm{A}$ comparison indicates that the temporal growth rates decrease for Froude numbers equal to infinity, 10, 5, and 2 as the jet proceeds downstream. The maximum growth rates decrease by about $7 \%$ for $\mathrm{Fr}=\infty$ and 10 , by about $6.4 \%$ for $\mathrm{Fr}=5$, and by about $2.3 \%$ for $\mathrm{Fr}=2$. At $\mathrm{Fr}=1$, however, the maximum growth rate increases by about $14 \%$ while the wavenumber corresponding to the maximum growth rate is decreased by about $8 \%$. As the Froude number is further reduced at this axial location, trends similar to those for $R / \theta=10$ are observed, except at $\mathrm{Fr}=1$ where there is a change in the wavenumber corresponding to the maximum growth rate.

Figures 8 and 9 display the variation of the phase velocities of the disturbances at various Froude numbers for $R / \theta=10$ and 5 respectively. At $R / \theta=10$, the influence of Froude number on the phase velocity, is to lower the phase velocities when compared to those of the constant density jet at most wavenumbers. But, at $\mathrm{Fr}=1$, for wavenumbers less than 0.02 the phase velocities are increased, and are even greater than 1. This suggests that disturbances at these low wavenumbers move with a velocity faster than the jet. 
Subbarao and Cantwell (1992) observed the formation of "secondary vortex-ring-like structures" at a Richardson number of 1.6 (equivalent to $\mathrm{Fr}=1.12$ ) that accelerated rapidly upward within the core fluid and increased the entrainment and breakdown of the jet. The increased phase velocities at low wavenumbers may be associated with these secondary vortexring-like structures. As the jet moves downstream from $R / \theta=$ 10 to $R / \theta=5$, the increased phase velocities are evident at $\mathrm{Fr}=$ 2 , with phase-velocities at $\mathrm{Fr}=1$ increasing further by about $18 \%$ for these low wavenumbers.

In order to understand the effect of buoyancy on the jet instability, the Froude number was reduced further to 0.2 for $R / \theta=10$ in Figures 10 and 11. It is seen that the growth rates and range of unstable wavenumbers are further increased with a reduction in Froude number. When $\mathrm{Fr}$ is reduced from infinity to 0.5 , the maximum growth rate is increased by about $74 \%$ and the range of unstable wavenumbers is increased by about $14 \%$, while the wavenumber corresponding to the maximum growth rate is decreased by about $8 \%$. The maximum growth rate and range of unstable wavenumbers are increased by $187 \%$ and $31 \%$ respectively when Fr is reduced to 0.3 from infinity, while the wavenumber corresponding to the maximum growth rate is increased by about $8 \%$. Reducing $\mathrm{Fr}$ from infinity to 0.2 results in a $350 \%$ increase in the maximum growth rate, $55 \%$ increase in the range of unstable wavenumbers and a $46 \%$ increase in the wavenumber corresponding to the maximum growth rate. Thus for $\mathrm{Fr}<1$, the growth rates are dramatically increased, signifying that the jet becomes highly unstable for buoyancy-driven jet flows. This observation is in agreement with the experiments conducted by Cetegen and Kasper (1996).

In Figure 11, the phase velocities are plotted as a function of wavenumber. As $\mathrm{Fr}$ is reduced below 1 to 0.2 , the range of low wavenumbers corresponding to phase velocities greater than 1 is increased from 0.02 to 0.04 . Also, the maximum phase velocity is almost doubled at $\mathrm{Fr}=0.2$. These results can be understood in light of the extra term in equation (14) due to the presence of gravity. The non-dimensional coefficient of the buoyancy term in this equation is

$$
G=\frac{1}{F^{2} \frac{R}{\theta}\left(\frac{\rho_{\infty}}{\rho_{j}}-1\right)}=\frac{g}{\left(U_{j}^{2} / \theta\right)}
$$

$G$ is a non-dimensional number that compares the acceleration of the large-scale structures of the jet due to buoyancy to that due to inertial forces. The variation of $G$ with Froude number for $R / \theta=10$ and 5 is indicated in Figure 12 at a density ratio of 0.14 . At both $R / \theta=10$ and 5 , for Froude numbers less than $1, G$ increases almost exponentially. Also, the values of $G$ at $R / \theta=5$ are about double those at $R / \theta=10$. The increase in growth rates at $\mathrm{Fr}=1$ as the jet proceeds downstream from $R / \theta=10$ to $R / \theta=5$ is captured well by this variation in G. Thus, the effect of buoyancy at low Fr values is to cause the jet instability to increase dramatically. This could explain the reason for the abrupt breakdown of the jet largescale structures noticed in helium-in-air experiments by Subbarao and Cantwell (1992) and Cetegen and Kasper (1996).

\section{CONCLUSIONS}

In this study, the instability characteristics of a lowdensity round gas jet injected into a high-density ambient gas were investigated. A linear temporal stability analysis was performed to solve for eigenvalues of the pressure disturbance equation obtained from the simultaneous solution of the equations of motion. The effects of buoyancy and density ratio on the evolution of the disturbances with time were determined.

A reduction in the density ratio steepens the density gradient in the shear layer, causing additional disturbance. Thus the temporal growth rates of the disturbances increase as the density ratio is decreased and the range of unstable wavenumbers also increases. The wavenumbers corresponding to the maximum growth rates also increase with decreasing density ratio. As the Froude number is reduced, the disturbance growth rates increase and the range of unstable wavenumbers also increase. A new non-dimensional number that compares the acceleration of the large-scale structures of the jet due to buoyancy to that due to inertial forces was found to play a significant role in the instability characteristics. At low Froude numbers, buoyancy causes the jet instabilities to increase exponentially which accounts for the abrupt breakdown of the jet large-scale structures observed in experiments.

\section{ACKNOWLEDGMENTS}

This work was supported by a grant from the NASA Microgravity Fluid Physics program.

\section{REFERENCES}

Buckmaster, J. and Peters, N., (1986) "The Infinite Candle and its Stability - A Paradigm for Flickering Diffusion Flames," Twenty-First Symposium (International) on Combustion, pp. 1829-1836.

Cetegen, B. M. and Kasper, K. D., (1996) "Experiments on the Oscillatory Behavior of Buoyant Plumes of Helium and Helium-Air Mixtures," Physics of Fluids, Vol. 8, No 11, pp. 2974-2984.

Cetegen, B. M., (1997-1) "Behavior of Naturally Unstable and Periodically Forced Axisymmetric Buoyant Plumes of Helium and Helium-Air Mixtures," Physics of Fluids, Vol. 9, No 12, pp. 3742-3752.

Cetegen, B. M., (1997-2) "Measurements of Instantaneous Velocity Field of a Non-Reacting Pulsating Buoyant Plume by Particle Image Velocimetry," Combustion Science and Technology, Vol. 123, pp. 377-387.

Cetegen, B. M. and Dong, Y. (2000) "Experiments on the Instability modes of Buoyant Diffusion Flames and Effects of Ambient Atmosphere on the Instabilities," Experiments in Fluids, Vol. 28, pp. 546-558. 
Chen, L. D., Seaba, J. P., Roquemore, W. M., and Goss, L. P. (1988) "Buoyant Diffusion Flames," Twenty-Second Symposium (International) on Combustion, The Combustion Institute, pp. 677-684.

Cohen, J. and Wygnanski, I. (1987) "The Evolution of the Instabilities in the Axisymmetric Jet. Part 2. The Flow Resulting from the Interaction between Two Waves," Journal of Fluid Mechanics, Vol. 176, pp. 221.

Gebhart, B. (1993) Heat Conduction and Mass Diffusion, McGraw-Hill, New York.

Hamins, A., Yang, J. C. and Kashiwagi, T., (1992) “An Experimental Investigation of the Pulsation Frequency of Flames," Twenty-Fourth Symposium (International) on Combustion/The Combustion Institute, pp. 1695-1702.

Jendoubi, S. and Strykowski, (1994) "Absolute and Convective Instability of Axisymmetric Jets with External Flow," Physics of Fluids, Vol. 6, No. 9, pp. 3000-3009.

Kyle, D. M. and Sreenivasan, K. R., (1993) "The Instability and Breakdown of a Round Variable-Density Jet," Journal of Fluid Mechanics, Vol. 249, pp. 619-664.

Michalke, A. (1984) "Survey on Jet Instability Theory," Progress in Aerospace Sciences, Vol. 21, pp. 159-199.

Monkewitz, P. A. and Sohn, K. D. (1988) "Absolute Instability in Hot Jets," AIAA Journal, Vol. 26, pp. 911-916.

Pasumarthi, K. S., (2000) "Full Field Scalar Measurements in a Pulsating Helium Jet Using Rainbow Schlieren Deflectometry," MS Thesis, University of Oklahoma, Norman, Oklahoma.

Raynal, L., Harion, J.-L., Favre-Marinet, M. and Binder, G. (1996) "The Oscillatory Instability of Plane Variable-Density Jets," Physics of Fluids, Vol. 8, No. 4, pp. 993-1006.

Richards, C. D., Breuel, B. D., Clark, R. P. and Troutt, T. R., (1996) "Concentration Measurements in a Self-Excited Jet," Experiments in Fluids, Vol. 21, pp. 103-109.

Schlichting, H. (1979) Boundary Layer Theory, $7^{\text {th }}$ edition, McGraw-Hill, New York.

Subbarao, E. R. and Cantwell, B. J., (1992) "Investigation of a Co-Flowing Buoyant Jet: Experiments on the Effects of Reynolds Number and Richardson Number," Journal of Fluid Mechanics, Vol. 245, pp. 69-90.

Yu, M.-H. (1990) "Local and Global Instability of Heated 2-D Jets and Wakes," PhD Dissertation, University of California.

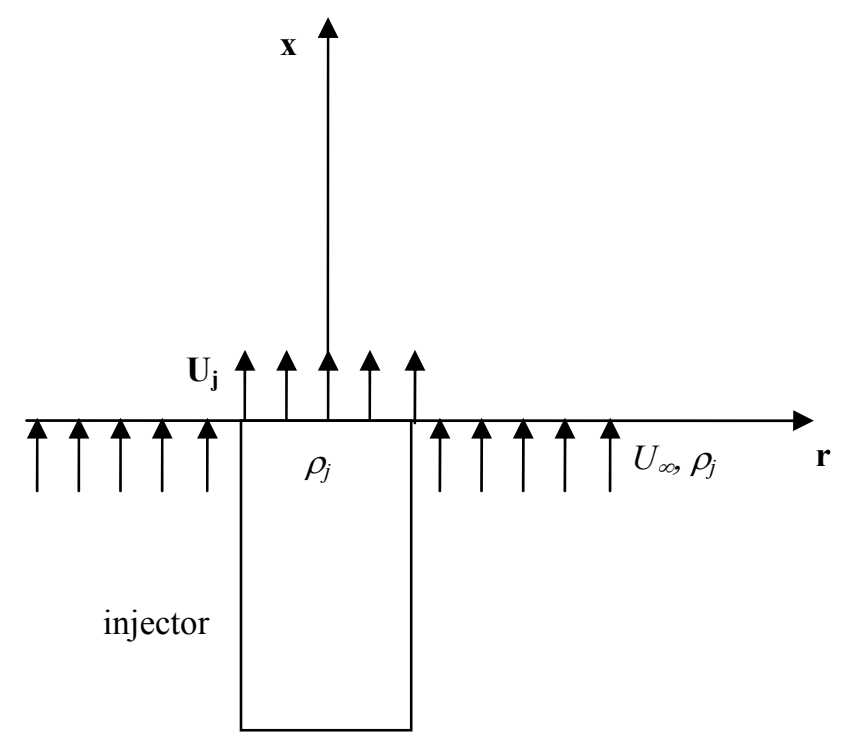

\section{Figure 1: Schematic of a low-density round gas jet injected vertically upwards into a high-density ambient gas}

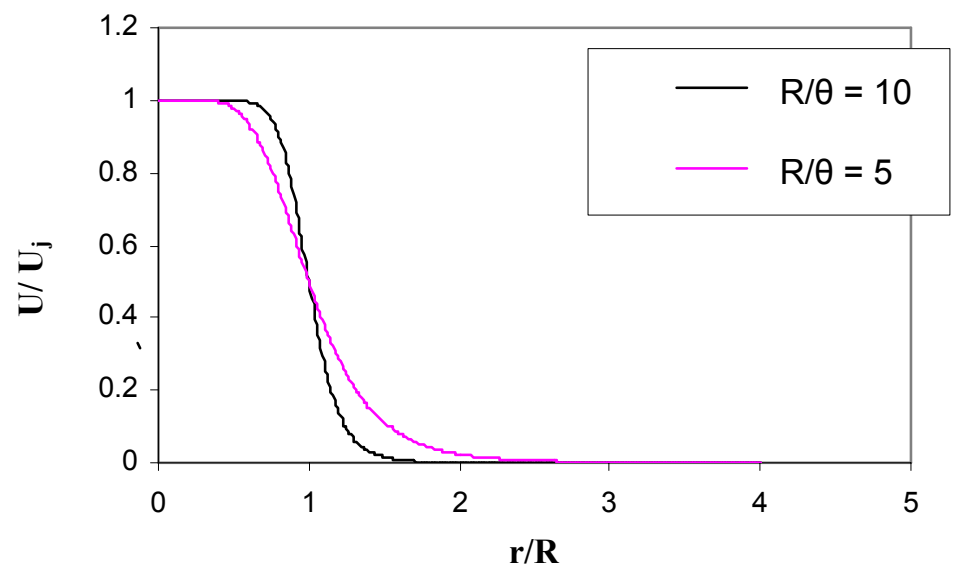

Figure 2: Nondimensionalized basic jet velocity profile for $S=1$ at $R / \theta=10$ and 5 




Figure 3: Density profile vs $r / R$ for $R / \theta=10$ at $S=1,0.6$ and 0.14



Figure 4: Temporal growth rate $\Omega_{i}$ as a function of wavenumber $\mathbf{k}$ for $\mathbf{m}=\mathbf{0}$, $R / \theta=10, F r=$ infinity, $S=1,0.7,0.6,0.5$, $0.4,0.3,0.2,0.14$ 


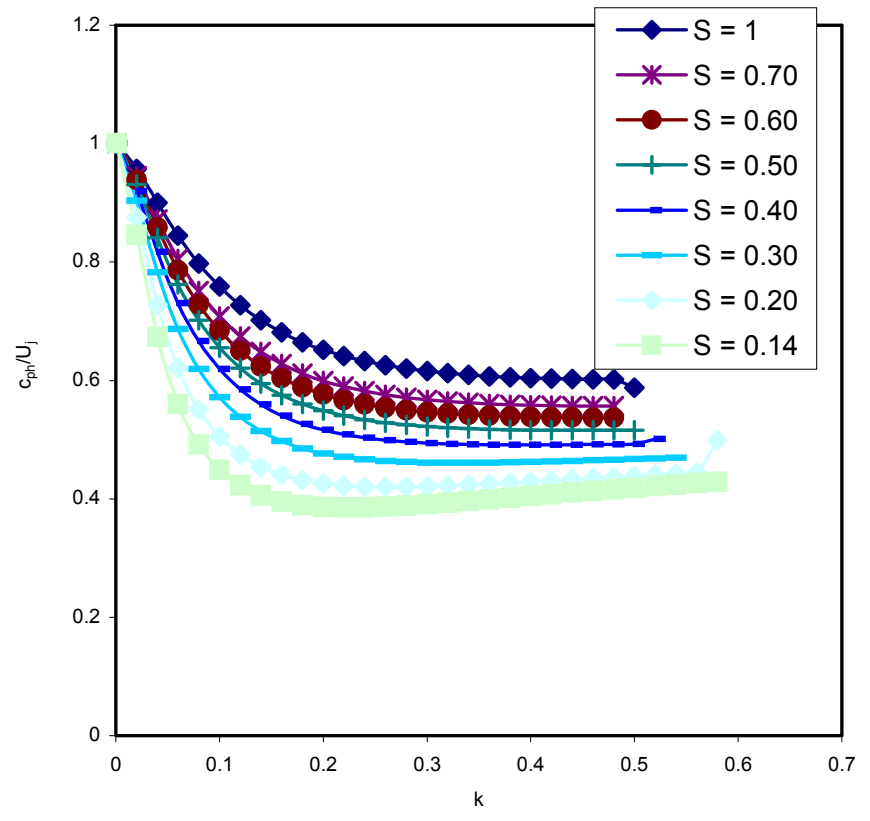

Figure 5: Phase velocity $c_{p h}$ as a function of wavenumber $\mathbf{k}$ for $\mathrm{m}=0, \mathrm{R} / \boldsymbol{\theta}=10$, $\mathrm{Fr}=$ infinity, $\mathrm{S}=1,0.7,0.6,0.5,0.4,0.3$, $0.2,0.14$

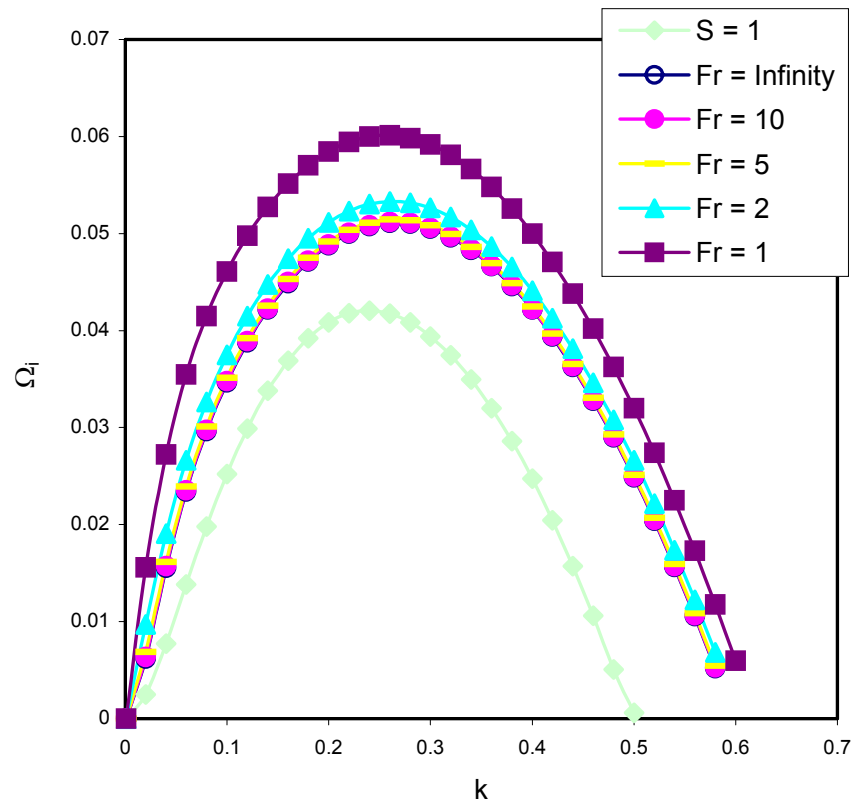

Figure 6: Temporal growth rate $\Omega_{\mathrm{i}}$ as a function of wavenumber $k$ for $m=0, R / \theta=10$, $S=1$ and $0.14, F r=$ Infinity, 10, 5, 2, 1

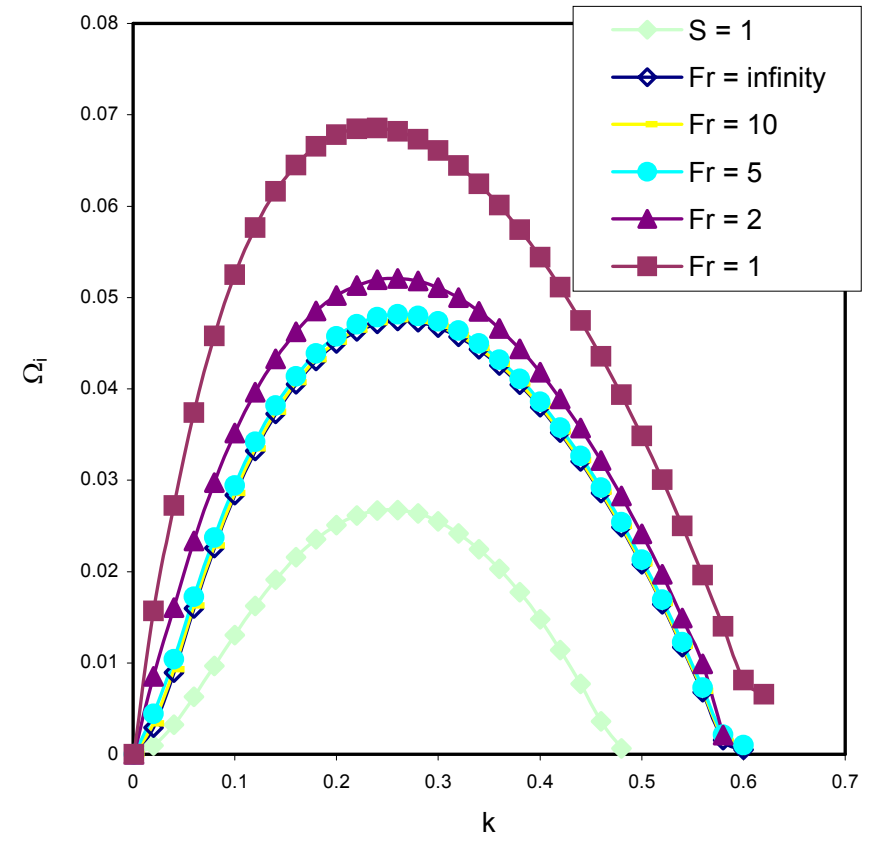

Figure 7: Temporal growth rate $\Omega_{\mathrm{i}}$ as a function of wavenumber $k$ for $m=0, R / \theta=5$, $S=1$ and $0.14, F r=$ Infinity, 10, 5, 2, 1

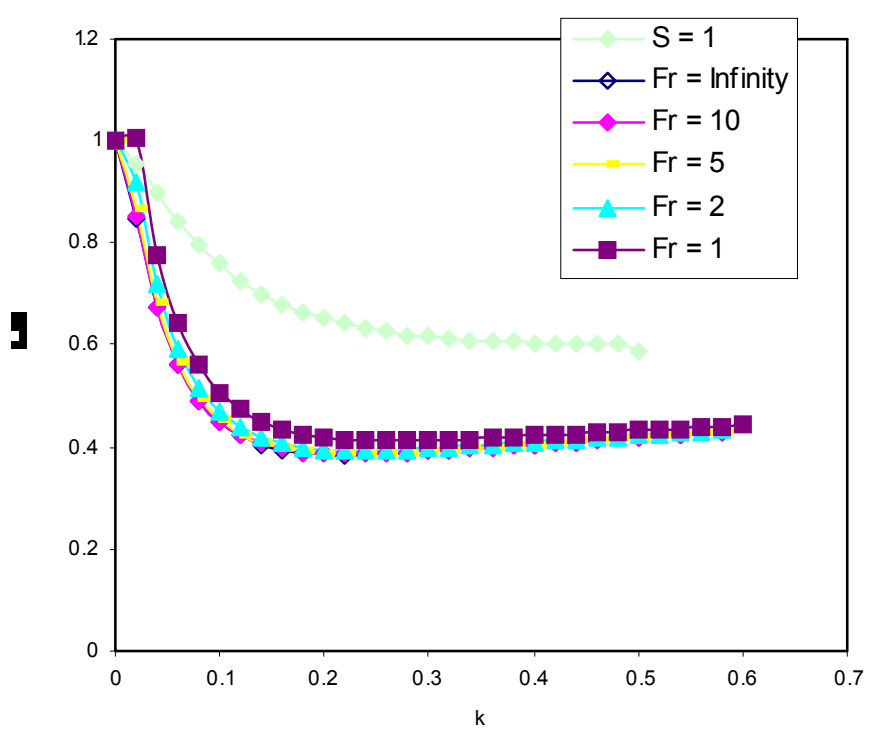

Figure 8: Phase velocity $\mathrm{c}_{\mathrm{ph}}$ as a function of wavenumber $\mathrm{k}$ for $\mathrm{m}=\mathbf{0}, \mathrm{R} / \boldsymbol{\theta}=10, \mathrm{~S}=1$ and $0.14, \mathrm{Fr}=$ Infinity, 10, 5, 2, 1 




Figure 9: Phase velocity $c_{p h}$ as a function of wavenumber $k$ for $m=0, R / \theta=5, S=1$ and $0.14, \mathrm{Fr}=$ Infinity, 10, 5, 2, 1

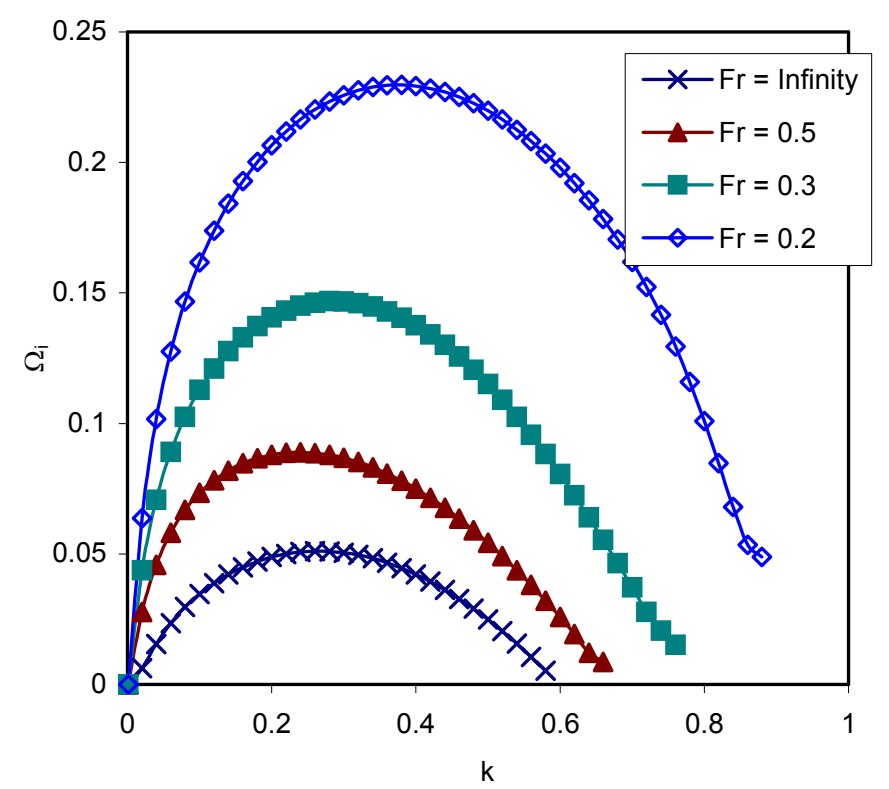

Figure 10: Temporal growth rate $\Omega i$ as a function of wavenumber $k$ for $m=0, R / \theta=10$, $S=0.14, \mathrm{Fr}=$ Infinity, 0.5, 0.3, 0.2



Figure 11: Phase velocity $\mathrm{c}_{\mathrm{ph}}$ as a function of wavenumber $\mathrm{k}$ for $\mathrm{m}=0, \mathrm{R} / \boldsymbol{\theta}=10, \mathrm{~S}=0.14$, $\mathrm{Fr}=$ Infinity, 0.5, 0.3, 0.2

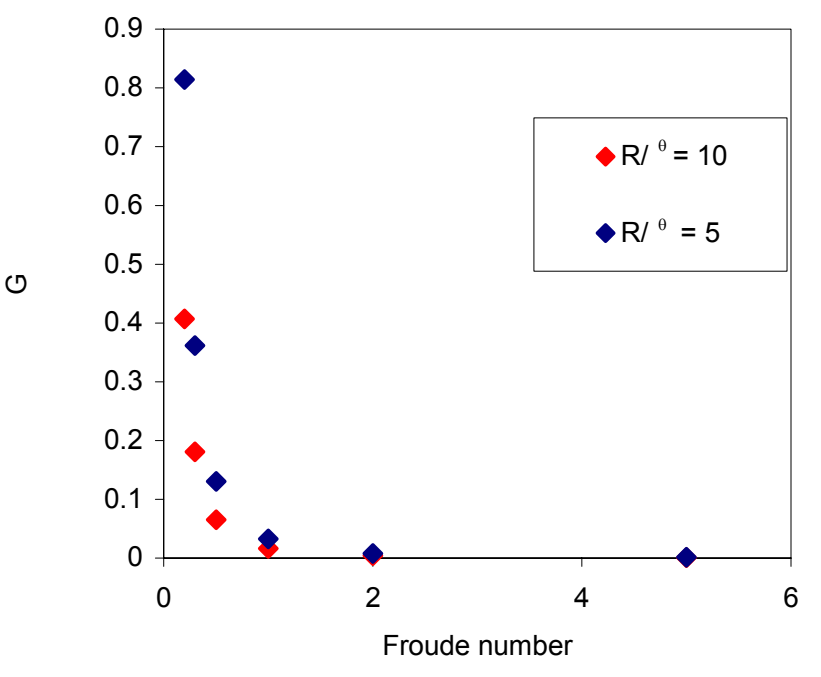

Figure 12: $\mathbf{G}$ vs Froude number for $\mathrm{S}=$ 0.14 at $R / \theta=10$ and 5 